\title{
Equatorial coronal holes, solar wind high-speed streams, and their geoeffectiveness ${ }^{\star}$
}

\author{
G. Verbanac ${ }^{1}$, B. Vršnak ${ }^{2}$, A. Veronig ${ }^{3}$, and M. Temmer ${ }^{3}$ \\ 1 Department of Geophysics, Faculty of Science, University of Zagreb, Horvatovac 95, 10000 Zagreb, Croatia \\ e-mail: verbanac@irb.hr \\ 2 Hvar Observatory, Faculty of Geodesy, University of Zagreb, Kačićeva 26, 10000 Zagreb, Croatia \\ 3 Institute for Physics, University of Graz, Universitätsplatz 5, 8010 Graz, Austria
}

Received 31 March 2010 / Accepted 9 October 2010

\begin{abstract}
Context. Solar wind high-speed streams (HSSs), originating in equatorial coronal holes (CHs), are the main driver of the geomagnetic activity in the late-declining phase of the solar cycle.

Aims. We analyze correlations between $\mathrm{CH}$ characteristics, HSSs parameters, and the geomagnetic activity indices, to establish empirical relationships that would provide forecasting of the solar wind characteristics, as well as the effect of HSSs on the geomagnetic activity in periods when the effect of coronal mass ejections is low.

Methods. We apply the cross-correlation analysis to the fractional $\mathrm{CH}$ area $(\mathrm{CH})$ measured between central meridian distances $\pm 10^{\circ}$, solar wind parameters (flow velocity $V$, proton density $n$, temperature $T$, and the magnetic field $B$ ), and the geomagnetic indices $D s t$ and $A p$.

Results. The cross-correlation analysis reveals a high degree of correlation between all studied parameters. In particular, we show that the $A p$ index is considerably more sensitive to HSS and CH characteristics than Dst. The $A p$ and $D s t$ indices are most tightly correlated with the solar wind parameter $B V^{2}$.

Conclusions. From the point of view of space weather, the most important result is that the established empirical relationships provide a few-days-in-advance forecasting of the HSS characteristics and the related geomagnetic activity at the six-hour resolution.
\end{abstract}

Key words. Sun: corona - solar-terrestrial relations - solar wind

\section{Introduction}

Solar wind high-speed streams (HSSs), originating from equatorial coronal holes $(\mathrm{CHs})$, and the associated co-rotating interacting regions (CIRs) formed in front of HSSs (e.g., Gosling 1996, and references therein) cause medium-level, long-lasting, recurrent geomagnetic activity (e.g., Nolte et al. 1976; Vršnak et al. 2007b, and references therein). The geomagnetic effects are caused by the southward component of the interplanetary magnetic field $\left(B_{\mathrm{s}}\right)$ related to Alfvénic fluctuations within CIR/HSS structures (e.g., Schwenn 1983; Tsurutani \& Gonzalez 1987; Tsurutani et al. 2004). Consequently, the most geoeffective region is expected to be the magnetic field compression within the CIR, where the field is strong and fluctuations are amplified.

Thus, forecasting the properties of HSS/CIRs is a central concern of space weather. Two methods are currently applied. The heliospheric MHD numerical codes like ENLIL (e.g., Lee et al. 2009; Odstrcil \& Pizzo 2009, and references therein), or empirical/numerical hybrid models (e.g., HHMS; Detman et al. 2006) predict the basic physical parameters of the solar-wind, using synoptic solar magnetic field data as the model input. On the other hand, purely empirical methods provide forecasting of the solar wind characteristics at the Earth by utilizing relationships between the $\mathrm{CH}$ characteristics and the properties of HSSs

\footnotetext{
* Appendices, Figs. 9-14, and table 4 are only available in electronic form at http://www. aanda.org
}

(Nolte et al. 1976; Robbins et al. 2006; Vršnak et al. 2007a; Luo et al. 2008; Abramenko et al. 2009; Obridko et al. 2009). The empirical approach also enables a direct forecasting of the geomagnetic activity (Vršnak et al. 2007b).

In this paper, we built upon the empirical method proposed by Vršnak et al. (2007a,b) by increasing the time "resolution" from one-day to six-hour intervals. In a preliminary analysis, we tested various options, and found that a six-hour resolution is the optimal one, because when shorter intervals are applied the results do not change much but the noise level increases. Furthermore, we extend the study by performing an analysis of various combined solar wind parameters, and beside the geomagnetic $D s t$ index we include also the $A p$ index.

Thus, there are two goals of the paper. The first is to analyze the correlation of indices $D s t$ and $A p$ with the HSS/CIR parameters (such as magnetic field $B$, velocity $V$, a measure of the convective electric field $V B$, and the Poynting flux $V B^{2}$ ) to learn in more detail how different magnetospheric current systems respond to the HSS/CIR-related solar-wind disturbances. The second is to prepare an empirical tool for predicting the HSS/CIR characteristics and forecasting the related geomagnetic activity.

In Sect. 2, we present the data. The correlations of the solar wind parameters with the geomagnetic indices are analyzed in Sect. 3, whereas in Sect. 4 we present a procedure that can be used for the forecasting of the HSS/CIR characteristics and the level of the geomagnetic activity based on $\mathrm{CH}$ measurements. The results are discussed in Sect. 5. Details of the 
cross-correlation analysis are provided in Appendix A, whereas in Appendix B we present some intriguing aspects of correlations between various solar wind parameters.

\section{Data}

Our study is based on the following data sets:

- solar wind parameters (density $n$, temperature $T$, velocity $V$, and the magnetic field strength $B$ );

- fractional area $C H$ of coronal holes;

- geomagnetic indices Dst and Ap.

We focus on a period in the declining phase of the solar cycle No. 23, when the coronal mass ejection (CME) activity was very low and the solar wind was modulated primarily by HSSs. As in the papers by Vršnak et al. (2007a,b) and Temmer et al. (2007), we consider the period from 25 January 2005 to 5 May 2005 (day-of-year, $D O Y=25-125$ ).

The solar wind data were obtained from the Solar Wind Electron Proton and Alpha Monitor (SWEPAM; McComas et al. 1998) and the magnetometer instrument (MAG; Smith et al. 1998) onboard the Advanced Composition Explorer (ACE; Stone et al. 1998) ${ }^{1}$.

Solar coronal-hole fractional areas were determined from soft X-ray images acquired by the Soft X-ray Imager (SXI; Hill et al. 2005; Pizzo et al. 2005) onboard the GOES-12 spacecraft ${ }^{2}$. For our purposes, we used four SXI coronal images per day taken around $0,6,12$, and $18 \mathrm{UT}$. In measuring the $\mathrm{CH}$ areas, we applied a fixed threshold to the calibrated SXI coronal hole images of $0.15 \mathrm{DN} \mathrm{s}^{-1}$. All pixels below this threshold (identified as coronal hole pixels) within a specified region of the solar disk were summed and divided by the total number of pixels of the considered region to evaluate the fractional area $\mathrm{CH}$ covered by coronal holes (for details, see Vršnak et al. 2007a). We determined the fractional area $\mathrm{CH}$ in the central-meridian slice, extending from the central meridian distance $-10^{\circ}$ to $+10^{\circ}$.

Planetary geomagnetic activity index $A p$ is a measure of the daily level of the mid-latitude magnetic activity. It is derived from a range of geomagnetic field variations over a period of $3 \mathrm{~h}$ from measurements provided by 13 geomagnetic observatories between $44^{\circ}$ and $60^{\circ}$ northern or southern geomagnetic latitudes ${ }^{3}$.

The storm-time disturbance index $D s t$, represents the axially symmetric disturbance magnetic field at the dipole equator on the Earth's surface. Any decrease in Dst is mainly due to the ring current, while positive variations in Dst are caused by the compression of the magnetosphere produced by the increase in solar wind dynamic pressure. The Dst index is derived from hourly scalings of the horizontal magnetic field variations measured at four low-latitude geomagnetic observatories ${ }^{4}$.

The six-hour running means of $D s t$ are evaluated by averaging hourly values in the range of $\pm 3 \mathrm{~h}$ centered at $0,6,12$, and 18 UT. We note that $A p$ index is given as the three-hourlyvalue, so the time window of of $\pm 3 \mathrm{~h}$ contains only three values for averaging.

\footnotetext{
${ }_{1}$ In particular, we used the merged hourly-averaged level-2 ACE data given at http://www.srl.caltech.edu/ACE/ASC/level2/

${ }^{2}$ In particular, we used the SXI coronal hole image product (level-2 files; see http://sxi.ngdc.noaa.gov/)

3 More details and data can be found at http://wdc.kugi. kyoto-u.ac.jp/cgi-bin/kp-cgi

${ }^{4}$ Explanations and data are available at http://swdcwww.kugi. kyoto-u.ac.jp/dstdir/
}

Time series of $C H, T, n, B, V, D s t$, and $A p$ are shown in Fig. 1. We performed the spectral analysis and found period of 9.1 days for all quantities (see also Crowley et al. 2008; Lei et al. $2008 a, b, c)$, which corresponds to $1 / 3$ of the solar synodic rotation period. The periodicity is a consequence of three long-lived equatorial $\mathrm{CHs}$ separated in longitude by $\approx 120^{\circ}$ (Vršnak et al. 2007a,b; Temmer et al. 2007).

In the analyzed period, these three coronal holes passed across the central meridian 11 times, resulting in $11 \mathrm{HSS} / \mathrm{CIR}$ structures at $1 \mathrm{AU}$ (enumerated in Fig. 1; see also Table 1 of Vršnak et al. 2007a). The CIRs are seen as the density and magnetic field bumps ahead of HSSs.

In Fig. 2, we present a close-up of HSS No. 11 (DOY = 118-125) to illustrate in more detail a typical relationship between the behavior of the geomagnetic activity indices and the HSS/CIR-related solar-wind properties. Figure 2 a shows that the Ap peaks and Dst dips appear at the frontal side of HSS (rise of the solar-wind speed), closely related to the CIR magnetic field compression. Moreover, the graph shows that the Ap peak appears during the decreasing phase of $D s t$, i.e., the $D s t$ dip is delayed with respect to the $A p$ peak. This is emphasized in Fig. $2 \mathrm{~b}$ where $A p(t)$ is compared to the change rate in Dst ( $\Delta D s t$ represents the change of $D s t$ over the 6-h intervals). The graph shows that, within the considered time resolution, the peaks of $A p$ and $\Delta D s t$ are contemporaneous (for a similar relationship between Dst and AE activity, see Gonzalez et al. 1994, and references therein). Furthermore, both peaks are contemporaneous with peaks of the product $V B$. This implies that $A p$ and $V B$ behave as a derivative of the $D s t$, or more accurately, that $D s t$ represents a cumulative consequence of $V B$, whereas $A p$ is a direct effect of $V B$. This behavior is more or less repeated in all HSS/CIRs in the analyzed period.

\section{Cross-correlations}

In the following, we investigate the relationship between various solar wind parameters $(n, B, T, V)$, fractional $\mathrm{CH}$ area $C H$, and the geomagnetic indices $A p$ and $D s t$, by applying the crosscorrelation analysis. All cross-correlation functions (presented in graphs given in Appendix A) are derived up to a time lag of \pm 10 days, with a step of $6 \mathrm{~h}$ (data resolution) in all investigated cases. The time lags are always expressed in days. Negative lag between two quantities, e.g., $V$ and $A p$, hereinafter denoted as the $V-A p$ correlation, means that $V$ is delayed with respect to $A p$.

\subsection{Cross-correlation between solar wind parameters and geomagnetic indices}

We calculated the cross-correlation functions for all combinations of solar-wind parameters and the geomagnetic indices $D s t$ and $A p$. The cross-correlation functions showing the highest correlation coefficients are presented in Appendix A.

In Figs. 3 and 4, the corresponding scatter plots for the highest-correlation time-lag are presented for the most tightly correlated parameters. The most relevant correlations are listed in Table 1 where the time lags $\Delta t$, the corresponding linear least squares fit parameters $a$ and $b$, and the correlation coefficient $R$ are presented. A given " $X-Y$ " correlation corresponds to the linear form $Y(t)=a X\left(t^{*}\right)+b$, where $X\left(t^{*}\right)$ represents the value of $X$ that occurred $\Delta t$ days before the true value of $Y(t)$, i.e., $t^{*}$ is the "retarded time", $t^{*}=t-\Delta t$.

Inspecting Table 1 one finds that in the case of the Dst index, the highest correlation coefficient $(R=0.58)$ is found 


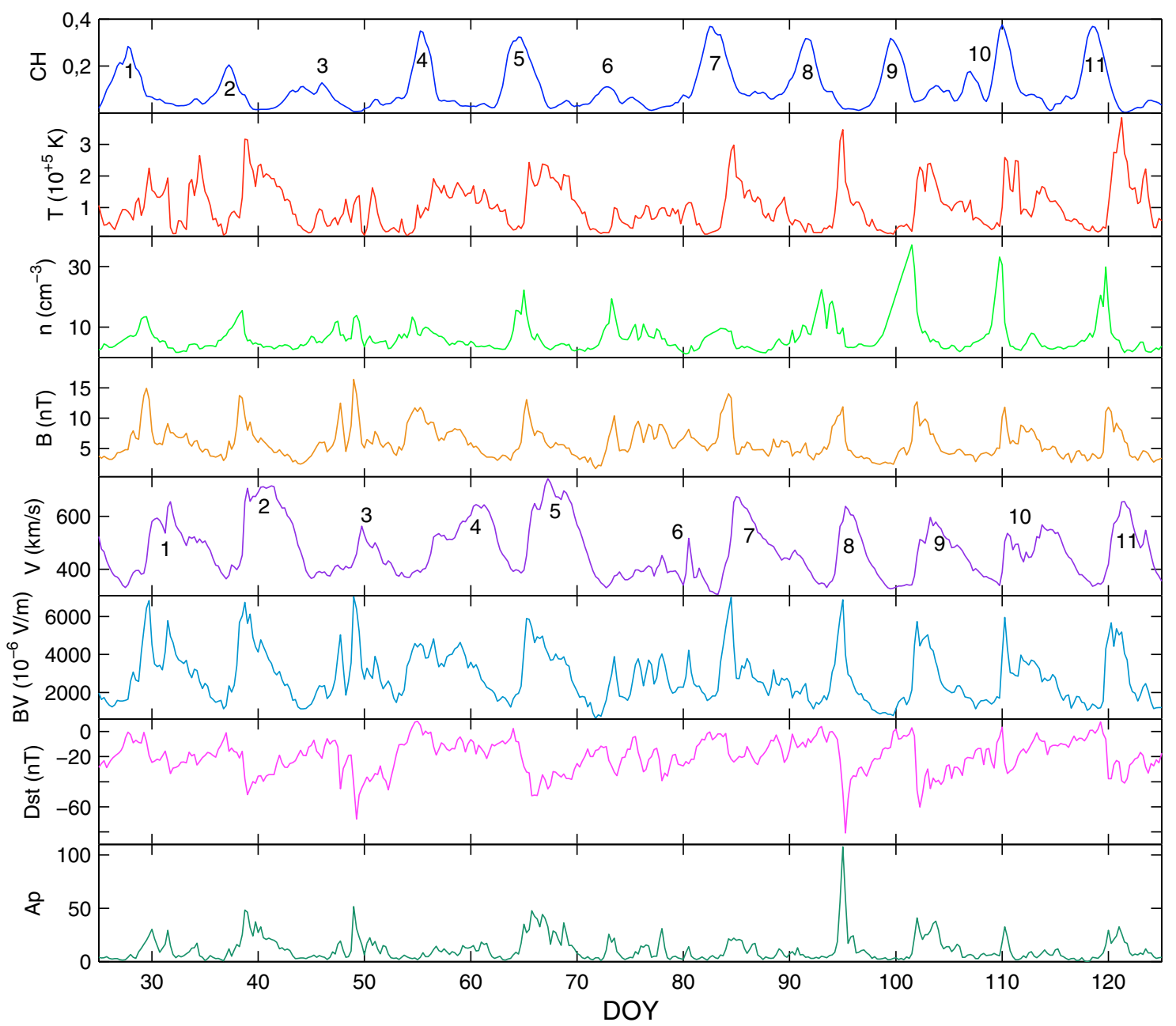

Fig. 1. Time series (6-h data resolution) of $C H$ (fractional area), $T(\mathrm{~K}), n\left(\mathrm{~cm}^{-3}\right), B(\mathrm{nT}), V\left(\mathrm{~km} \mathrm{~s}^{-1}\right), B V, D s t(\mathrm{nT})$, and $A p(\mathrm{nT})$, from top to bottom, respectively. The time is expressed in the day-of-year form, $D O Y$, for 2005.

between $D s t$ and $B V^{2}$, which are anti-correlated, with the time delay of $\Delta t=0.25 \mathrm{~d}$. Similar, but slightly lower, correlation coefficients are obtained for $V-D s t$ and $B V-D s t$ relationships. We note that the Dst dip is contemporaneous with the $V$ peak, whereas it is delayed with respect to all other solar wind parameters.

The data presented in Fig. 3 can be straightforwardly compared with the results presented by Richardson \& Cane (2010). We considered the total magnetic field, while they considered the southward component of the magnetic field, $B_{\mathrm{s}}$. They analyzed a set of geomagnetic storms caused by magnetic clouds and found a high degree of correlation between $D s t$ and solar wind parameters $B_{\mathrm{s}}, V$, and $B_{\mathrm{s}} V(R=0.89,0.54$, and 0.90 , respectively; the corresponding regression lines are drawn by dotted lines) ${ }^{5}$. The slopes of $\operatorname{Dst}\left(B_{\mathrm{s}}\right)$ and $\operatorname{Dst}\left(B_{\mathrm{s}} V\right)$ found by Richardson \& Cane (2010) are steeper than our $D s t(B)$ and $D s t(B V)$ slopes, which is to be expected, since $B_{\mathrm{s}}<B$ : the regression lines from Richardson \& Cane (2010) are close to the lower limit edge of our $D s t(B)$ and $D s t(B V)$ data scatter. On the other hand, the steeper $\operatorname{Dst}(V)$ slope in Richardson \& Cane (2010) cannot be explained in this manner; it indicates that CIRs affect the geomagnetic field in a somewhat different manner than CMEs: at the same flow speed, CMEs are far more effective.

\footnotetext{
5 Note that the unit for the product $B V$ used in our graphs, $\mathrm{nT} \times \mathrm{km} \mathrm{s}^{-1}$, corresponds to $0.001 \mathrm{mV} \mathrm{m}^{-1}$.
}

In addition, our CIR Dst- $V$ correlation can be compared with the $D s t-V$ correlations of MCs and non-MCs as reported by Gopalswamy (2010). He studied the major storms of solar cycle 23 and found that the CMEs resulting in MCs exibit a strong $D s t-V$ correlation $(R=-0.72)$, while CMEs that cause non-MCs exibit a very weak correlation $(R=-0.14)$. For CIR storms analysed in our study, the $D s t-V$ correlation $(R=0.54)$ is weaker than for MCs, but much stronger than for non-MCs.

Interestingly, for the intense CIR storms within the cycle 23, Gopalswamy (2008) found a much weaker correlation between both $V$ and $B V$, and $D s t(R<0.3)$.

The data-point distribution in Fig. $3 \mathrm{~b}$ shows that the moderate storm level ( $D$ st below $-50 \mathrm{nT}$ ) can be achieved only if magnetic field is strong enough, i.e., it has to be stronger than $5 \mathrm{nT}$. A storm can become intense $(D s t<-100 \mathrm{nT})$ only if $B>10 \mathrm{nT}$. These values are consistent with previous estimates of the lower limit to the southward component of the magnetic field $B s$ (see Gonzalez et al. 1994, and references therein).

When comparing the magnetic content of CIRs with the MC and non-MC magnetic content, Gopalswamy (2008) found that the total magnetic field strength for CIRs, MCs and non-cloud ICMEs is as high as $19 \mathrm{nT}, 23 \mathrm{nT}$ and $20 \mathrm{nT}$, respectively (see Fig. 12 in his work). The magnetic content of CIRs in our study reaches the maximum value of $16 \mathrm{nT}$.

For the $A p$ index, the strongest correlation is found is between $A p$ and $B V^{2}$, where $\Delta t=0$ and the correlation coefficient 

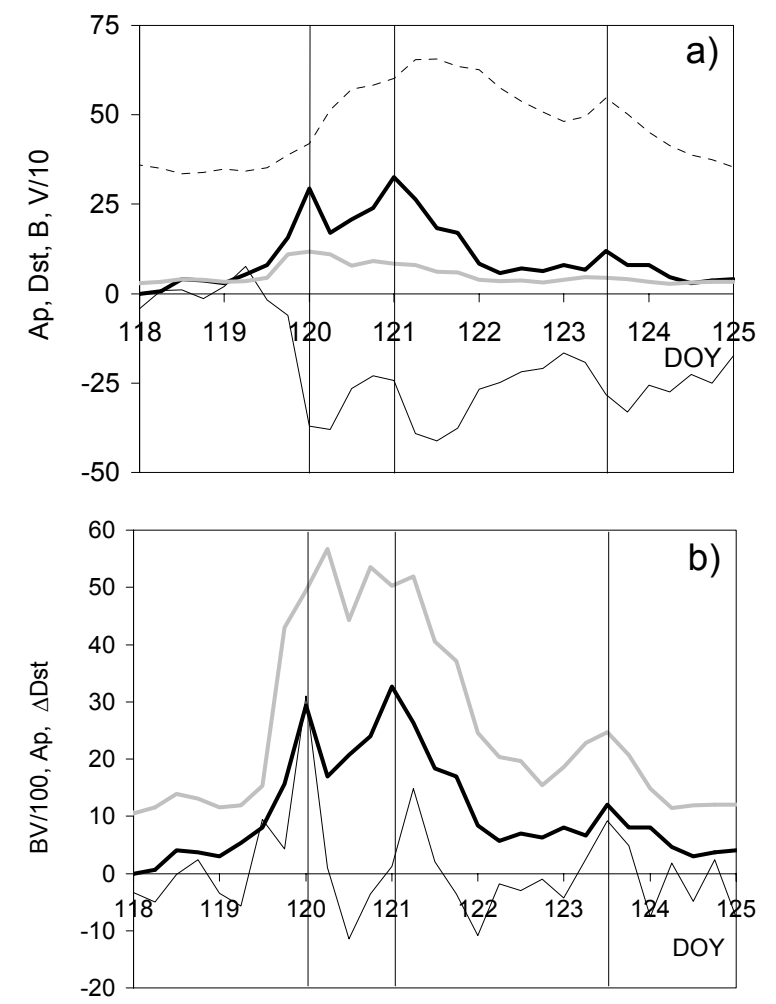

Fig. 2. Close-up of the interval DOY $=118-125$ (HSS No. 11): a) development of $A p[\mathrm{nT}]$ (bold-black) and $D s t[\mathrm{nT}]$ (thin-black) compared to the variation in the solar wind speed $V\left[\mathrm{~km} \mathrm{~s}^{-1} / 10\right]$ (dashed) and the magnetic field $B$ [nT] (bold-gray); b) $A p$ (bold-black) compared to the change rate of $D s t(\Delta D s t$, thin-black) and the product $B V$ (bold-gray).

$R=0.77$. The peak of $A p$ is also contemporaneous with the peak of $B V$ and $B^{2} V$. Finally, we fitted the $A p(V)$ dependence using a quadratic function of the form $A p=a V^{2}+b$ (see, Crooker et al. 1977), and we got $A p=7 \times 10^{-5} V^{2}-5.4$, which is quite close to the relationship found by Crooker et al. (1977) for the yearly-averaged values. We note that the difference between the correlation coefficients of the linear and quadratic fit is not significant (there being $R=0.66$ and $R=0.67$, respectively).

The data in panels a) - c) of Figs. 3 and 4 are presented separately for $v>450 \mathrm{~km} \mathrm{~s}^{-1}$ (circles) and $v<450 \mathrm{~km} \mathrm{~s}^{-1}$ (black dots). Comparing the correlations for the case $v>450 \mathrm{~km} \mathrm{~s}^{-1}$ (check the inset in Figs. 3 and 4) with those obtained for the complete sample (Table 1), one finds that the correlation coefficients remain at more or less the same level. However, the slopes of regression lines become steeper. On the other hand, for $v<450 \mathrm{~km} \mathrm{~s}^{-1}$ data, the correlation coefficients become significantly lower and the slope of regression lines are less steep. This clearly indicates that high speed streams are more geoeffective than either fluctuations within the slow solar wind or frontal parts of corotating interacting regions where the wind speed is still low. In other words, magnetic field compressions and higher wind speed are both needed to enhance the geomagnetic activity.

Indices $A p$ and $D s t$ are anti-correlated with $R=0.75$ for the delay of $D s t$ after $A p$ of $\Delta t=0.25$ (Fig. 5 and the last row of Table 1). Although the correlation-coefficient is high, we note that these two indices show considerable differences. For example, $D s t$ can attain values as low as $-40 \mathrm{nT}$, without practically any signal in $A p$ index.

The explanation can be found in the different behavior of the $A p$ and $D s t$ variations (see, Fig. 2), namely that the $D s t$ variation is characterized by the recovery phase whose duration is longer
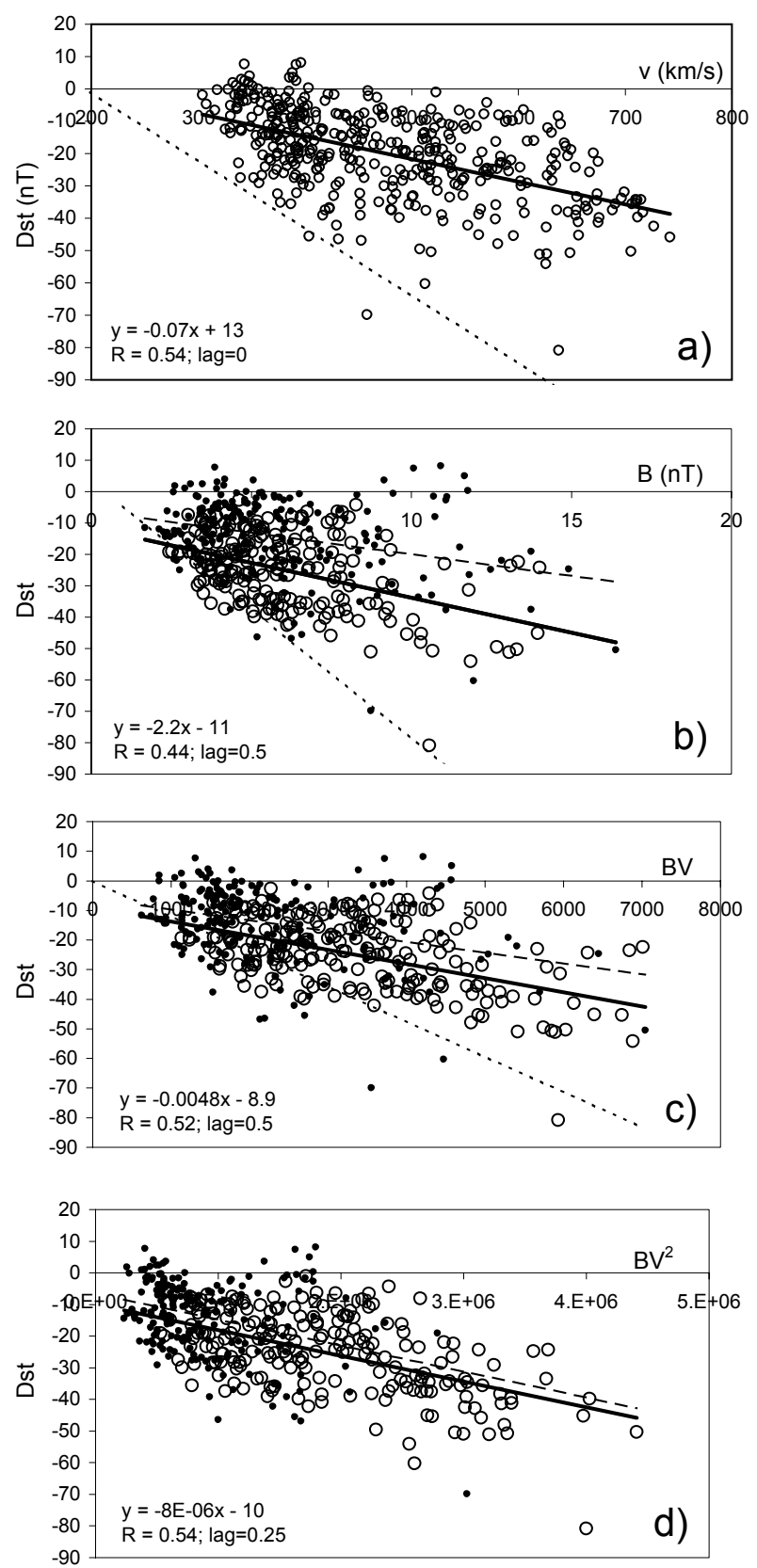

Fig. 3. Correlations $V-D s t, B-D s t, B V-D s t$, and $B V^{2}-D s t$. In panels b)-d) we separated the data points characterized by $v>450 \mathrm{~km} \mathrm{~s}^{-1}$ (circles), from those having $v<450 \mathrm{~km} \mathrm{~s}^{-1}$ (black dots). The linear least squares fit parameters, the correlation coefficient $R$, and the applied time lag $\Delta t$ are shown in the insets; in panels b)-d) the fit parameters are shown only for the $v>450 \mathrm{~km} \mathrm{~s}^{-1}$ data (bold lines); dashed lines represent the fits for the $v<450 \mathrm{~km} \mathrm{~s}^{-1}$ data, dotted lines represent the results from Richardson \& Cane (2010).

than the duration of the $A p$ peak. Thus, although the $A p$ index already attains low values, the $D s t$ is still quite far from $D s t=0$.

The difference can also be seen by comparing Figs. 3 and 4 , which have a different types of data scatter. Inspecting graphs in Fig. 4, we find that the distribution of data-points exibit a triangular pattern, where the lower limit of $A p$ is approximately aligned with $A p=0$ for a wide range of values characterizing the solar-wind parameters ( $x$-axis). On the other hand, the upper limit of $A p$ is clearly dependent on the solar-wind parameters. For example, the upper limits can be expressed approximately 

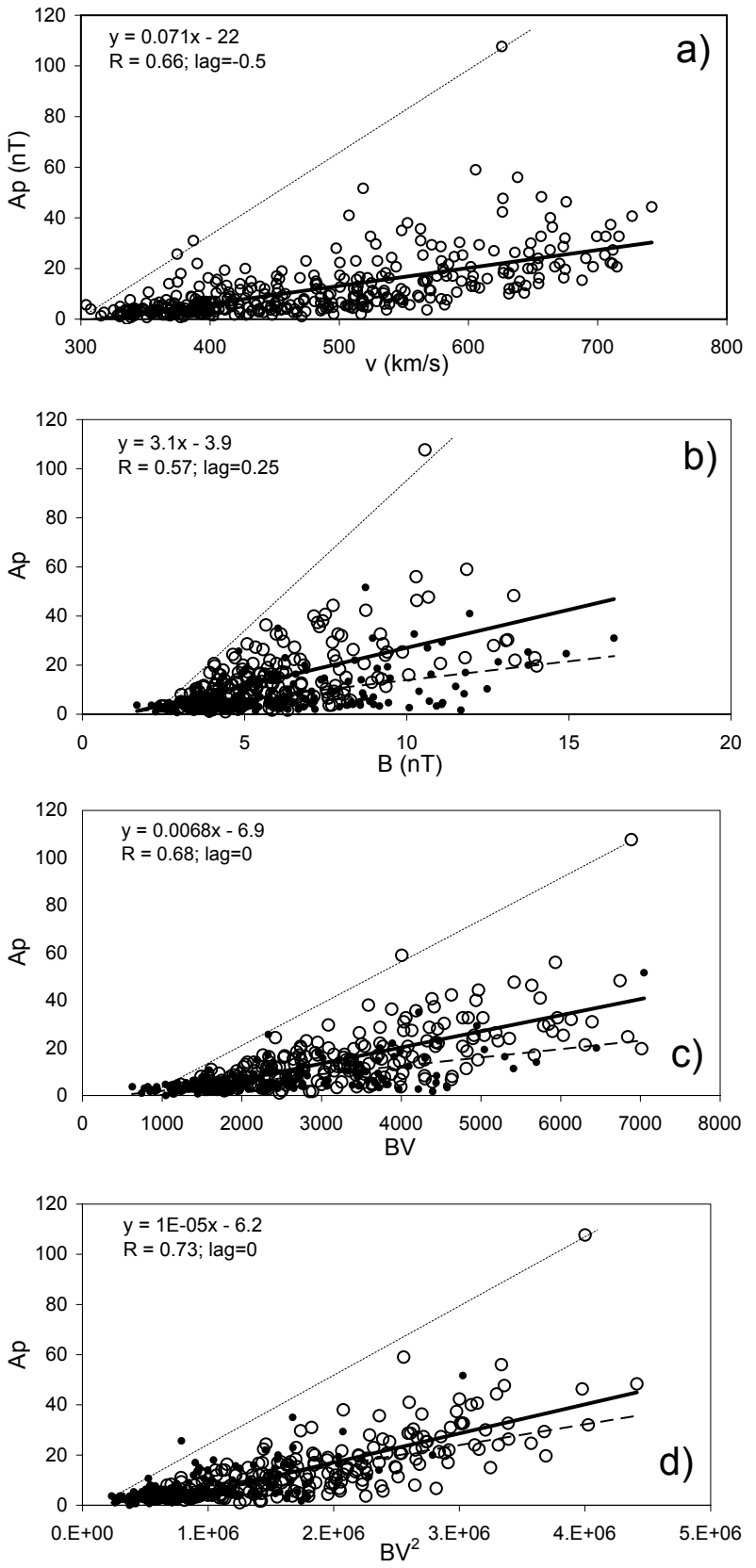

Fig. 4. Correlations $V-A p, B-A p, B V-A p$, and $B V^{2}-A p$. In panels b)-d) we separated the data points characterized by $v>450 \mathrm{~km} \mathrm{~s}^{-1}$ (circles), from those having $v<450 \mathrm{~km} \mathrm{~s}^{-1}$ (black dots). The linear least squares fit parameters, the correlation coefficient $R$, and the applied time lag $\Delta t$ are presented in the insets; in panels $\mathbf{b})-\mathbf{d}$ ), the fit parameters are for the $v>450 \mathrm{~km} \mathrm{~s}^{-1}$ data (bold line). Dashed lines are fits for the $v<450 \mathrm{~km} \mathrm{~s}^{-1}$ data; dotted lines represent an estimate of the upper limit.

as $A p \approx 0.3(V-300), A p \approx 12(B-2), A p \approx 6(B V-500)$, and $A p \approx 3\left(B V^{2}-0.2\right)$, where $V$ is expressed in $\mathrm{km} \mathrm{s}^{-1}$ and $B$ in $\mathrm{nT}$.

The described "triangular distribution" of data points in Fig. 4 means that large values of $V, B, B V$, or $B V^{2}$ do not necessarily imply that a high level of geomagnetic activity is beeing measured by $A p$. On the other hand, high values of $A p$ cannot correspond to low values of the afore mentioned solar-wind parameters. In contrast to $A p$, the $D s t$-related correlations presented in Fig. 3 do not show such a pronounced "triangular" shape, i.e., the upper limit $(D s t=0)$ is not so sharply defined.

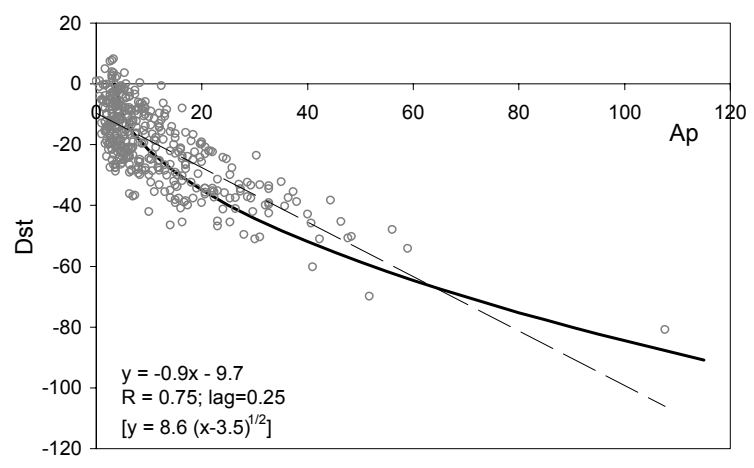

Fig. 5. Correlation between $D s t$ and $A p$, where $D s t$ is delayed by 0.25 days. Parameters of the linear least square fit (dashed line) are given in the inset. Bold curve represents the function Dst = $-8.6 \sqrt{A p-3.5}$.

Table 1. Relationships between geomagnetic indices and solar wind parameters.

\begin{tabular}{lrrrr}
\hline \hline & $a$ & $b$ & $R$ & $\Delta t$ \\
\hline$V-D s t$ & -0.07 & 13.0 & -0.52 & 0.00 \\
$B-D s t$ & -1.8 & -9.0 & -0.35 & 0.50 \\
$B V-D s t$ & -0.005 & -5.1 & -0.52 & 0.50 \\
$B^{2} V-D s t$ & $-3.2 \times 10^{-4}$ & -13.0 & -0.43 & 0.5 \\
$B V^{2}-D s t$ & $-9.3 \times 10^{-6}$ & -6.7 & -0.58 & 0.25 \\
$B^{2} V^{2}-D s t$ & $-7.3 \times 10^{-7}$ & -12.5 & -0.51 & 0.50 \\
\hline$V-A p$ & 0.07 & -22.2 & 0.66 & -0.50 \\
$B-A p$ & 2.3 & -2.2 & 0.52 & 0.25 \\
$B V-A p$ & 0.0059 & -5.5 & 0.7 & 0.00 \\
$B^{2} V-A p$ & $3.7 \times 10^{-4}$ & 3.8 & 0.61 & 0.00 \\
$B V^{2}-A p$ & $1.0 \times 10^{-5}$ & -3.1 & 0.77 & 0.00 \\
$B^{2} V^{2}-A p$ & $8.7 \times 10^{-7}$ & 2.7 & 0.72 & 0.00 \\
\hline$A p-D s t$ & -0.9 & -9.7 & -0.75 & 0.25 \\
\hline
\end{tabular}

Notes. Linear least squares fit coefficients and the time lags $\Delta t$ for the relationships between geomagnetic indices and various solar wind parameters.

Finally, the distribution of data points in Fig. 5 indicates that the relationship $D s t(A p)$ might not be linear. Thus, we also tried a quadratic fit to the $A p(D s t)$ data, and the correlation coefficient increased to $R=0.83$, with $A p=0.015 D s t^{2}+0.078 D s t+4$. The same correlation coefficient was found for the reduced quadratic fit, $A p=0.013 D s t^{2}+3.5$ (corresponding to $D s t=$ $-8.6 \sqrt{A p-3.5}$, shown in Fig. 5). This implies that $A p$ can be represented as a quadratic function of $D$ st.

\subsection{The relationship to coronal holes}

The cross-correlation functions for the relationships between coronal hole fractional area, $\mathrm{CH}$, and the solar wind parameters are shown in Appendix A.

The corresponding scatter plots are shown in Fig. 6, and the relationships are summarized in Table 2, where we show the linear least squares fit parameters, the correlation coefficients $R$, and time lags $\Delta t$. The coronal hole fractional area is more tightly correlated with the solar wind velocity $(R=0.59$ and $\Delta t=3.75$ days).

This result agrees with earlier findings of Krieger et al. (1973), Neupert \& Pizzo (1974), Nolte et al. (1976). In the study by Nolte et al. (1976), a very high correlation (0.96) between coronal hole area and maximal velocity in the associated stream 

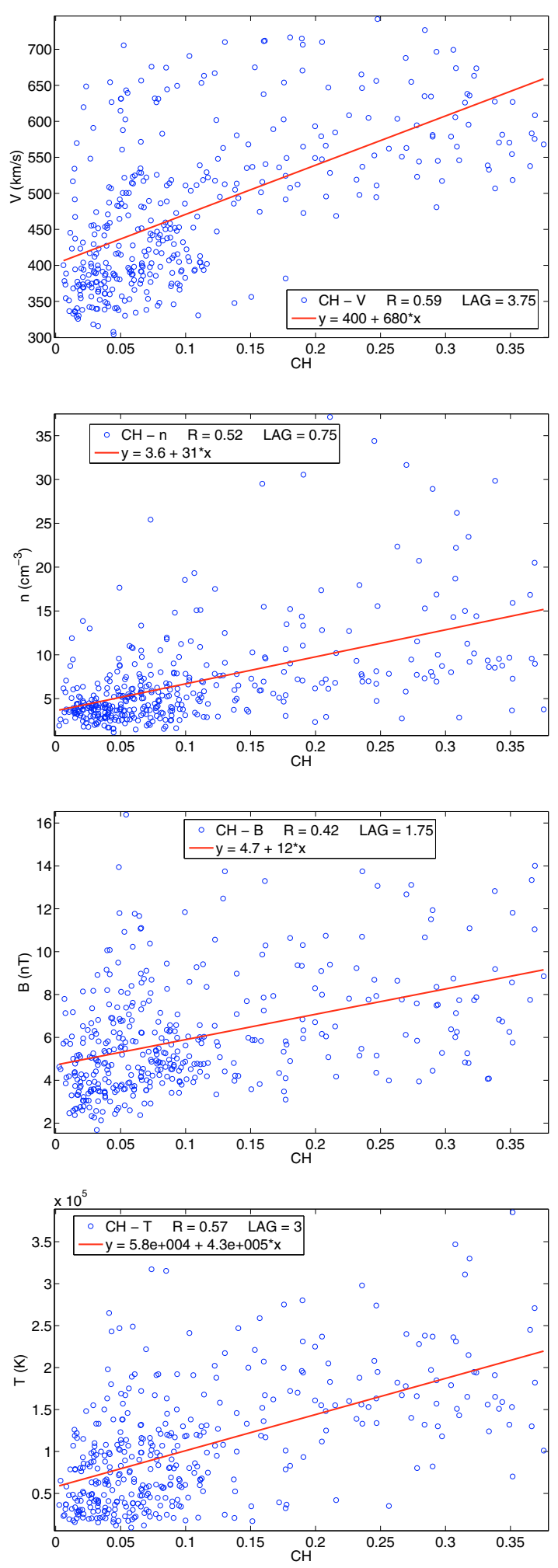

Fig. 6. Correlations $\mathrm{CH}-\mathrm{V}, \mathrm{CH}-n, \mathrm{CH}-\mathrm{B}$, and $\mathrm{CH}-\mathrm{T}$. The linear least squares fit parameters, correlation coefficient $R$, and the applied time lag $\Delta t$ are presented in the insets.

was obtained. The larger scatter in the data presented in our Fig. 6 (top panel), is due to: (a) usage of the fractional coronal hole area in the central meridian slice, not the entire $\mathrm{CH}$ area; (b) solar wind speed depends not only on the coronal hole size, but also on the magnetic field strength and the magnetic flux expansion rate (Fujiki et al. 2005).
Table 2. Relationships between solar wind parameters and $\mathrm{CH}$.

\begin{tabular}{lrrrrrr}
\hline \hline & $a$ & $b$ & $R$ & $\Delta t$ & $a_{\text {corr }}$ & $b_{\text {corr }}$ \\
\hline$C H-n$ & 31 & 3.6 & 0.52 & 0.75 & 60 & 2.5 \\
$C H-B$ & 12 & 4.7 & 0.42 & 1.75 & 29 & 3.5 \\
$C H-T$ & $4.3 \times 10^{5}$ & $5.8 \times 10^{4}$ & 0.57 & 3.00 & $8.1 \times 10^{5}$ & $1.5 \times 10^{4}$ \\
$C H-V$ & 680 & 400 & 0.59 & 3.75 & 930 & 350 \\
\hline
\end{tabular}

Notes. Linear least squares fit coefficients and the time lags $\Delta t$ for the relationships: $n(\mathrm{CH}), B(\mathrm{CH}), T(\mathrm{CH})$, and $V(\mathrm{CH})$. The "amplitudeoptimized" coefficients are denoted as $a_{\text {corr }}$ and $b_{\text {corr }}$.

Inspecting Table 2, we find that the average delay between the $C H(t)$ signal and the CIR-related $n(t)$ variation is only 0.75 days (consistent with the results presented by Vršnak et al. 2007a). At first sight, this might be surprising since the solarwind transit time is expected to be at least 2-3 days. A similar result is found for the estimated delay of $B(t)$, where $\Delta t=1.75 \mathrm{~d}$. To explain such short delays we have to take into account the spatial extent of coronal holes as well as the CIR-compression having formed at the frontal side of the HSS (Gosling 1996), i.e., it maps on to the leading edge of the coronal hole. From Fig. 1, one can see that the rise in the $C H(t)$ signal from the beginning to the peak lasts from 1 to 3 days. In other words, the leading edge of the $\mathrm{CH}$ has passed over the central meridian 1-3 days earlier than its central regions, which is a result of the large spatial extent of CHs. Thus, for the largest $\mathrm{CHs}$, at the moment when the "center" of $\mathrm{CH}$ passes over the central meridian, the $\mathrm{CH}$ leading edge is already quite far to the west. In these cases, the bump in $n$ can be almost contemporaneous with the peak of $C H$ (e.g., CIR No. 6 around $D O Y=73$; see also Fig. 1 in Vršnak et al. 2007a). In the case of a small CH, the delay tends to be longer, generally around 2-3 days. Thus, we examined the delays of $n$ bumps relative to the onset of the rise in the $\mathrm{CH}$ signal, and found out that the delays range between 2 and 4 days, with the average value of $3.6 \pm 1.1$ days.

In this respect, we note that sometimes, depending on the shape of the $\mathrm{CH}$ area, the $C H(t)$ signal displays a complex structure (see e.g., the double-peaked $\mathrm{CH}$ bump related to HSS No. $10, D O Y=107-110)$. This probably results in a complex evolution of the HSS/CIR structure in the interplanetary space, which may result in an apparently unexpected, early appearance of the CIR-related density compression.

Figure 7 shows the correlations $\mathrm{CH}-\mathrm{Dst}$ and $\mathrm{CH}-\mathrm{Ap}$ (the corresponding cross-correlation functions are shown in Appendix A). The time lags and the linear least squares fit parameters are given in Table 3 . We note that the correlation between $A p$ and $C H$ is much tighter than between $D s t$ and $C H$ $(R=0.43$ and $R=-0.27$, with $\Delta t=3.0$ and $\Delta t=3.75$, respectively).

\section{Forecasting the solar wind parameters and geomagnetic indices}

Linear regression coefficients $a$ and $b$ given in Table 3 can be employed to predict the solar wind parameters from the $\mathrm{CH}$ data in periods of low CME activity. In this respect, we note that since the linear regression analysis is based on the least squares procedure, its application leads to an underestimate of the predicted variation-amplitudes (see thin black line in Fig. 8). The main cause of this effect is the timing mismatch of some of the peaks in the two curves (for details see Vršnak et al. 2007a). 

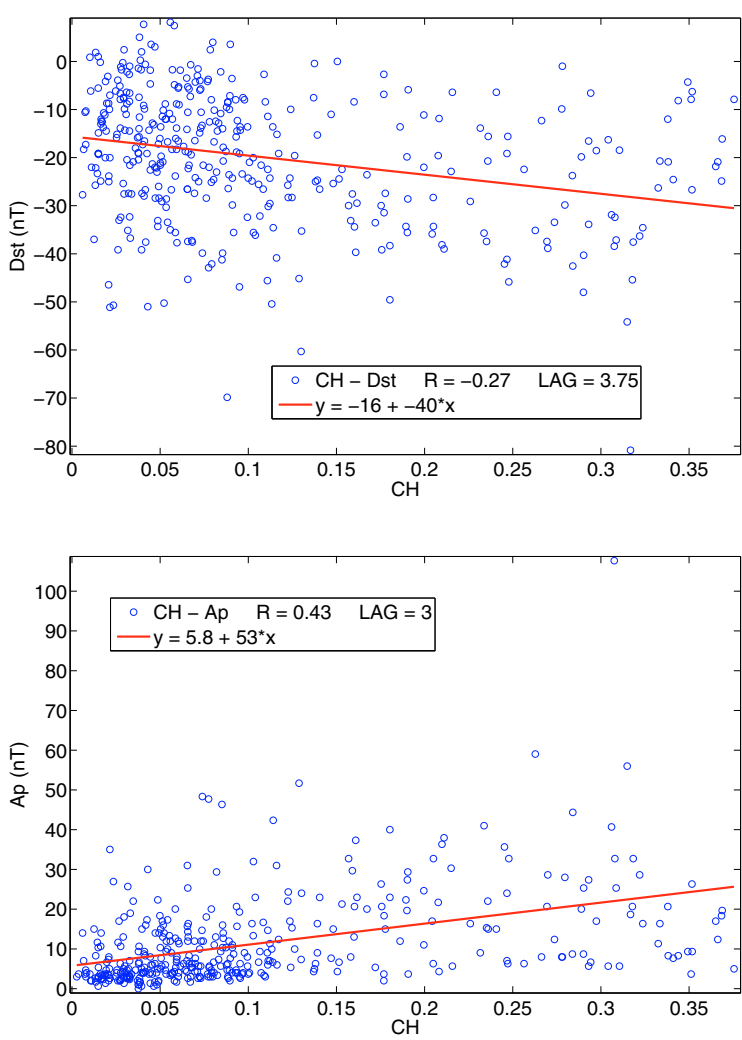

Fig. 7. Correlations $C H-D s t$ and $C H-A p$; the linear least squares fit parameters, the correlation coefficient $R$, and the applied time lag $\Delta t$ are presented in the insets.

Table 3. Relationship between $\mathrm{CH}$ and geomagnetic indices.

\begin{tabular}{lrrrr}
\hline \hline & $a$ & $b$ & $R$ & $\Delta t$ \\
\hline CH-D st & -40 & -16 & -0.27 & 3.75 \\
$C H-A p$ & 53 & 5.8 & 0.43 & 3.00 \\
\hline
\end{tabular}

Notes. Linear least squares fit coefficients and the time lags $\Delta t$ for the relationship between $\mathrm{CH}$ and geomagnetic indices.

Thus, since we are mainly interested in reproducing the real amplitudes (peak values), we subjectively "corrected" the linear regression coefficients to fit more closely the amplitudes themselves. In this procedure, it is necessary to decrease the value of parameter $b$, so that the "background" slow solar wind comes closer to the observed values, as well to increase parameter $a$ in order to stretch the amplitudes (see Figs. 8a-d).

Thus, we did not directly apply the relationships based on the linear least squares fit (parameters $a$ and $b$ as given in Table 2), but used them as a starting point for finding the most appropriate values of these parameters. After trying different combinations, we determined the optimal parameters/relationships that more properly reproduce the amplitudes. These parameters are given in Table 3 and are denoted as $a_{\text {corr }}$ and $b_{\text {corr }}$. Curves obtained with these coefficients are shown as a thick black curve in Figs. 8a-d.

The empirical relationship between the $\mathrm{CH}$ fractional areas and indices $D s t$ and $A p$ also opens the possibility of predicting the level of the HSS-related geomagnetic activity a few days in advance. In Figs. $8 \mathrm{e}-\mathrm{f}$, we present the observed values of $D s t$ and $A p$ (bold-gray line) and the values calculated using the relationships

$D s t=(-80 \pm 30 \times \cos \lambda) \times\left[C H\left(t^{*}\right)\right]^{1 / 2}$ and

$A p=(120 \pm 60 \times \cos \lambda) \times\left[C H\left(t^{*}\right)\right]$

(for details regarding Dst predictions see, Vršnak et al. 2007b), where, $\lambda$ is the ecliptic longitude and \pm applies as plus for $\mathrm{CHs}$ of positive magnetic polarity and as minus for negative polarity CHs. For forecasting purposes, $\lambda$ can be estimated using $\lambda=$ $2 \pi(t-81) / 365$, where the time $t$ is expressed as $D O Y$ (for details see, Vršnak et al. 2007b). The "predicted" values of geomagnetic indices are based on the $\mathrm{CH}$ areas measured at the retarded time $t^{*}=t-\Delta t$, where $\Delta t=3.75$ and $\Delta t=3$ are assumed for $D s t$ and $A p$, respectively.

The employed "forecast" relationships differ from those proposed by Vršnak et al. (2007a,b) because of the higher amplitude values found in our 6-h data resolution, which are smoothed in 24-h data used in their study.

\section{Discussion and conclusions}

We have analyzed the relationship between solar wind parameters, coronal hole areas $C H$, and geomagnetic $D s t$ and $A p$ indices for the period of low CME activity. The results are summarized as follows:

1. With 6-h (1/4 day) data resolution, we obtained good correlations between all studied parameters. This allows us to evaluate average time delays $\Delta t$ more accurately than in Vršnak et al. (2007a). A higher time resolution results in too large amounts of noise, so we consider the 6-h resolution as the optimal one.

2. $A p$ and $D s t$ are most tightly correlated with the solar-wind parameter-combination $B V^{2}(R=0.77$ and $R=-0.58$, respectively). The corresponding delays are $\Delta t=0$ days and $\Delta t=0.25$. The indices $A p$ and $D s t$ are correlated with $R=-0.75$, Dst being delayed for $\Delta t=0.25$ days.

3. The relationship $A p(D s t)$ can be represented by a quadratic dependence, with $D s t$ being delayed on average for 0.25 days. The peaks of $A p$ are contemporaneous with the peaks of the product $B V$, whereas at the same time $D s t$ displays the most sharpest decrease towards minimum.

4. Values of $\mathrm{Ap}$ are more strongly correlated with $\mathrm{CH}$ then $\mathrm{Dst}$ ( $R=0.43$ and -0.27 , respectively). The $D s t$ dip lags after the $C H$ peak for 3.75 days, while the $A p$ peak appears three days after the $\mathrm{CH}$ peak.

5. We have developed a procedure that allows a several-daysin-advance prediction of the solar wind parameters near the Earth, as well as the level of the HSS/CIR-related geomagnetic activity, from the $\mathrm{CH}$ observations, at a six-hour resolution.

The tightest correlation between both considered geomagnetic indices, $A p$ and $D s t$, is found with $B V^{2}$. The $A p\left(B V^{2}\right)$ relationship is quite similar to the relationship found by Crooker et al. (1977) for the yearly-averaged data. This behavior shows that the combination of the solar-wind speed and magnetic field is essential to the process of energy transfer from the solar wind to the magnetosphere.

The physical mechanism behind the $A p\left(B V^{2}\right)$ relationship is not entirely clear. For example, Ahluwalia (2000) noted that the physical unit for $B V$ corresponds to the force per unit charge and that of $B V^{2}$ to the power per unit charge. However, this does not explain the physical background of processes that lead to the situation where the geomagnetic activity indices have the tightest correlation with the product $B V^{2}$. 

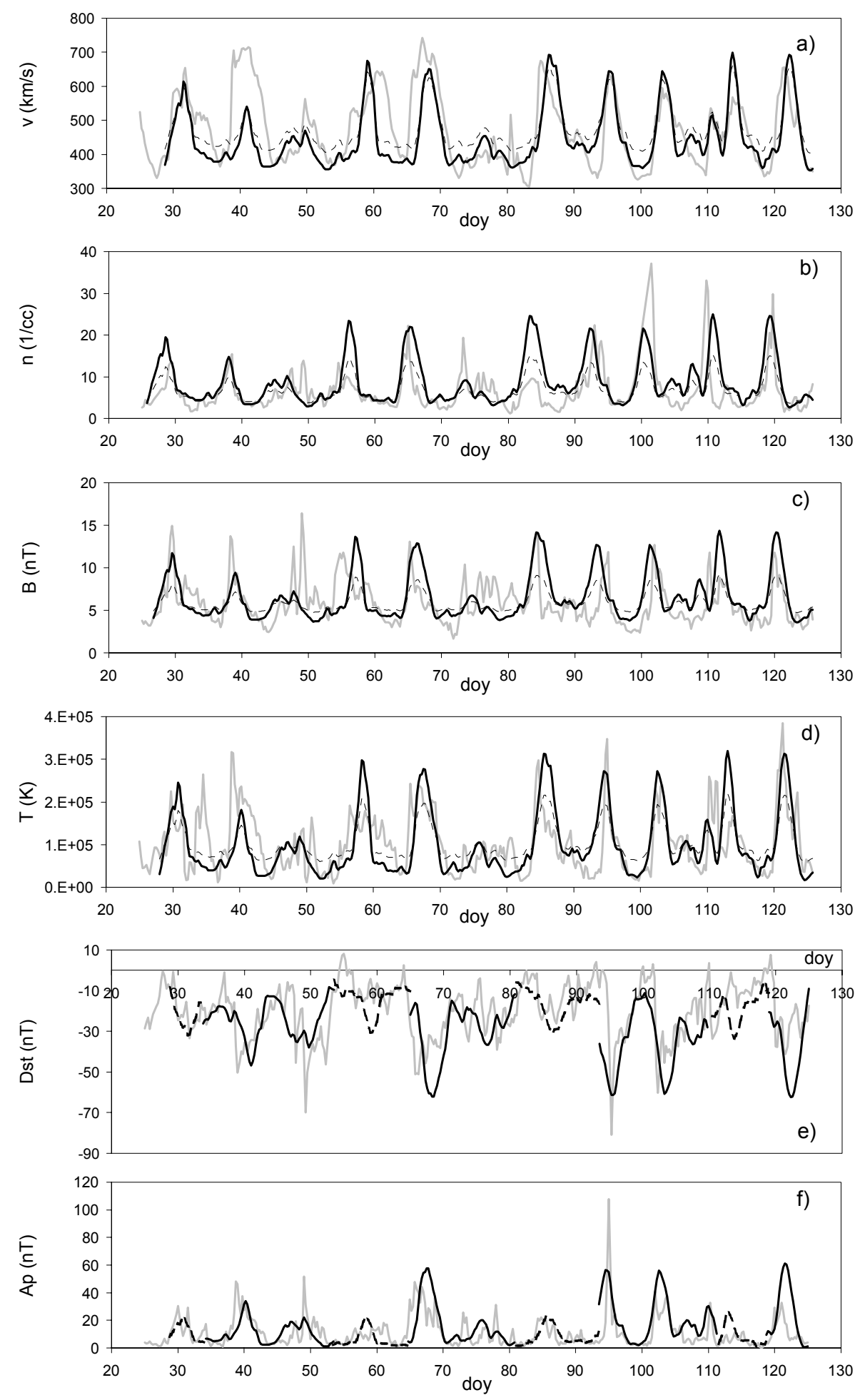

Fig. 8. a)-d) Comparison of the observed solar wind parameters with the calculated values. The observed data are inicated by the thick gray line, calculated values (using linear regression parameters $a$ and $b$ as given in Table 2) are drawn by thin black line, and the amplitude-optimized "predicted" values (using linear regression parameters $a_{\text {corr }}$ and $b_{\text {corr }}$ as given in Table 2) are drawn by thick black line. e)-f) Comparison of the measured values of the geomagnetic indices $D s t$ and $A p$ with the calculated values. The observed data are delineate by the thick gray line, values calculated for the $\mathrm{N}$-polarity $\mathrm{CHs}$ are drawn by thick-dashed line, and S-polarity CHs are indicated by thick-black line. See text for details.
According to the basic consideration of the energy transfer from the solar wind to the magnetosphere, the geomagnetic activity is forced by the magnetic reconnection near the nose of the magnetosphere (Siscoe \& Crooker 1974). Thus, it is clear that the product $B V$ related to the electric field in the current sheet, as well as the Poynting flux $V B^{2}$, both play an important role in determining the level of the geomagnetic activity (e.g., Perreault \& Akasofu 1978; Kan \& Lee 1979, and references therein).

In this respect, we note that Kan \& Lee (1979) have shown that the power delivered by the "solar wind dynamo" is proportional to $B^{2} V^{2}$. Our data indeed show that the $A p(B V)$ relationship, when expressed in the form $A p=a(B V)^{2}+$ $b$, has the correlation coefficient of $R=0.72$ at $\Delta t=0$ (Table 2). For the analogous relationship with $D s t$, we get $R=0.51$ at $\Delta t=+0.5$.

However, this still does not explain the dominant correlation of geomagnetic indices with the parameter $B V^{2}$, which obviously requires an additional explanation. One possibility is that this parameter represents the product of the induced electric field $B V$ and the merging velocity $V$. The first term determines 
the electric field in the current sheet that accelerates particles, whereas the $V$ term is related to the flow of particles entering into the current sheet.

The $A p$ index has a stronger correlation with all studied parameters than $D s t$ does, i.e., it reacts to HSSs more directly than $D s t$ (see also Verbanac et al. 2010). This implies that $A p$ is more sensitive to Alfvénic fluctuations in the solar wind highspeed streams (Tsurutani et al. 1995) than the Dst index.

Finally, we note that as a side result of our analysis we found two empirical invariants in the solar wind parameters. The value of $V^{2}$, multiplied by the density $n$ measured one day earlier, is approximately constant. Similarly, the solar wind speed $V$, multiplied by the magnetic field $B$ measured 2.25 days earlier, is also approximately constant. Although at the moment we cannot offer a physical explanation, we consider this finding as potentially very important for understanding of the time/spatial structure of the high-speed streams.

Acknowledgements. The research leading to the results presented in this paper has received funding from European Community's Seventh Framework Programme (FP7/2007-2013) under grant agreement No. 218816. We are grateful to GOES-SXI and ACE teams for their open data policies. We are thankful to the referee whose constructive comments led to significant improvement of the paper.

\section{References}

Abramenko, V., Yurchyshyn, V., \& Watanabe, H. 2009, Sol. Phys., 260, 43 Ahluwalia, H. S. 2000, J. Geophys. Res., 105, 27481

Crooker, N. U., Feynman, J., \& Gosling, J. T. 1977, J. Geophys. Res., 82, 1933

Crowley, G., Reynolds, A., Thayer, J. P., et al. 2008, Geophys. Res. Lett., 35, 21106

Detman, T., Smith, Z., Dryer, M., et al. 2006, J.Geophys. Res., 111, A07102

Fujiki, K., Hirano, M., Kojima, M., et al. 2005, Adv. Space Res., 35, 2185
Gonzalez, W. D., Joselyn, J. A., Kamide, Y., et al. 1994, J. Geophys. Res., 99, 5771

Gopalswamy, N. 2008, J. Atmos. Solar-Terrestr. Phys., 70, 2078

Gopalswamy, N. 2010, in IAU Symp., 264, 326

Gosling, J. T. 1996, ARA\&A, 34, 35

Hill, S. M., Pizzo, V. J., Balch, C. C., et al. 2005, Sol. Phys., 226, 255

Kan, J. R., \& Lee, L. C. 1979, Geophys. Res. Lett., 6, 577

Krieger, A. S., Timothy, A. F., \& Roelof, E. C. 1973, Sol. Phys., 29, 505

Lee, C. O., Luhmann, J. G., Odstrcil, D., et al. 2009, Sol. Phys., 254, 155

Lei, J., Thayer, J. P., Forbes, J. M., Sutton, E. K., \& Nerem, R. S. 2008a, Geophys. Res. Lett., 35, 10109

Lei, J., Thayer, J. P., Forbes, J. M., et al. 2008b, J. Geophys. Res., 113, A12, A11303

Lei, J., Thayer, J. P., Forbes, J. M., et al. 2008c, Geophys. Res. Lett., 35, L19105

Luo, B., Zhong, Q., Liu, S., \& Gong, J. 2008, Sol. Phys., 250, 159

McComas, D., Bame, S. J., Barker, P., et al. 1998, Space Sci. Rev., 86, 563

Neupert, W. M., \& Pizzo, V. 1974, in BAAS, 6, 292

Nolte, J. T., Krieger, A. S., Timothy, A. F., et al. 1976, Sol. Phys., 46, 303

Obridko, V. N., Shelting, B. D., Livshits, I. M., \& Asgarov, A. B. 2009, Sol. Phys., 260, 191

Odstrcil, D., \& Pizzo, V. J. 2009, Sol. Phys., 250, 297

Perreault, P., \& Akasofu, S. 1978, Geophys. J. R. Astron. Soc., 54, 547

Pizzo, V. J., Hill, S. M., Balch, C. C., et al. 2005, Sol. Phys., 226, 283

Richardson, I. G., \& Cane, H. V. 2010, Sol. Phys., 264, 189

Robbins, S., Henney, C. J., \& Harvey, J. W. 2006, Sol. Phys., 233, 265

Schwenn, R. 1983, Space Sci. Rev., 34, 85

Siscoe, G., \& Crooker, N. 1974, Geophys. Res. Lett., 1, 17

Smith, C. W., L'Heureux, J., Ness, N. F., et al. 1998, Space Sci. Rev., 86, 613

Stone, E. C., Frandsen, A. M., Mewaldt, R. A., et al. 1998, Space Sci. Rev., 86, 1

Temmer, M., Vršnak, B., \& Veronig, A. M. 2007, Sol. Phys., 241, 371

Tsurutani, B. T., \& Gonzalez, W. D. 1987, Planet. Space Sci., 35, 405

Tsurutani, B. T., Gonzalez, W. D., Gonzalez, A. L. C., et al. 1995, J. Geophys. Res., 100, 21717

Tsurutani, B. T., Gonzalez, W. D., Guarnieri, F., et al. 2004, J. Atmos. Solar-Terrestr. Phys., 66, 167

Verbanac, G., Vršnak, B., Temmer, M., Mandea, M., \& Korte, M. 2010, J. Atmos. Solar-Terrestr. Phys., 72, 607

Vršnak, B., Temmer, M., \& Veronig, A. 2007a, Sol. Phys., 240, 315

Vršnak, B., Temmer, M., \& Veronig, A. 2007b, Sol. Phys., 240, 331

Pages 10 to 13 are available in the electronic edition of the journal at http://www . aanda. org 


\section{Appendix A: Cross-correlation functions}

In Figs. 9-13, we present the cross-correlation functions between different parameters, which we used to perform the correlation scatter plots presented in the paper. All cross-correlation functions are derived up to a time lag of \pm 10 days, with a step of $6 \mathrm{~h}$ (data resolution) in all investigated cases. The time lags are always expressed in days. Negative lag between two quantities, e.g., $V$ and $A p$, hereinafter denoted as the $V-A p$ correlation, means that $V$ is delayed with respect to $A p$.

\section{Appendix B: Correlation between solar-wind parameters}

We calculated the cross-correlation functions for all combinations of $V, T, n$, and $B$. In Figs. 9 and 14, the crosscorrelation functions and the scatter plots for the highestcorrelation-coefficient time-lag are presented for the most tightly correlated combinations. All considered correlations are listed in Table 4 where the time lags $\Delta t$, the corresponding linear least squares fit parameters $a$ and $b$, and the correlation coefficient $R$ are presented. A given " $X-Y$ " correlation corresponds to the linear form $Y(t)=a X\left(t^{*}\right)+b$, where $X\left(t^{*}\right)$ represents the value of $X$ that occurred $\Delta t$ days before the actual value of $Y(t)$, i.e., $t^{*}$ is the "retarded time", $t^{*}=t-\Delta t$.

Table 4 shows that the most tightly correlated parameters are $V$ and $T$, having the correlation coefficient $R=0.8$, when $V$ is delayed after $T$ for $\Delta t=0.25$ days. Furthermore, Fig. 9 shows that in the $V-n$ and $V-B$ case, the correlation coefficients are higher when the parameters are anti-correlated (the negative correlation coefficient in Table 4). This reflects the main physical property of HSSs, which is a depleted density and magnetic field in the stream itself. The anti-correlations are most significant for the time lags $\Delta t=0.75 \mathrm{~d}$ and $\Delta t=2.25 \mathrm{~d}$, respectively, meaning that the dips of $B$ and $n$ are delayed with respect to the peak of $V$. The positive correlations of both the $V-B$ and $V-n$ relationships and their negative time lags (peaks of $n$ and $B$ preceding the peak of $V$ by $2.5 \mathrm{~d}$ and $1.75 \mathrm{~d}$ ), are related to the magnetic field and density compression in the "interacting region" at the frontal edge of HSSs. We note that the time lag in the $n-B$ correlation is $\Delta t=+0.5 \mathrm{~d}$, meaning that the peak of $B$ is delayed with respect to the peak in $n$.
Table 4. Relationships $T(V), n(V), B(V), T(B), B(n)$, and $T(n)$.

\begin{tabular}{lrrrr}
\hline \hline Relationship & $a$ & $b$ & $R$ & $\Delta t$ (days) \\
\hline$V-T$ & 530 & $-1.5 \times 10^{5}$ & 0.80 & -0.25 \\
$V-n$ & -0.03 & 21 & -0.56 & 0.75 \\
$V-B$ & -0.013 & 12 & -0.54 & 2.25 \\
$B-T$ & $1.5 \times 10^{4}$ & $1.6 \times 10^{4}$ & -0.44 & -2.50 \\
$n-B$ & 0.21 & 4.5 & 0.46 & 0.50 \\
$n-T$ & 600 & $1.4 \times 10^{5}$ & -0.48 & -1.00 \\
\hline
\end{tabular}

Notes. Linear least squares fit coefficients and the time lags $\Delta t$ for the relationships: $T(V), n(V), B(V), T(B), B(n)$, and $T(n)$. The expression used between two parameters $\left(X\right.$ and $Y$ ) is $Y(t)=a X\left(t^{*}\right)+b$, where $t^{*}$ is the "retarded time" $\left(t^{*}=t-\Delta t\right)$. The correlation coefficient is denoted as $R$.

The distribution of data points in $V-n$ and $V-B$ graphs in Figs. 14b and c shows two "branches", one almost horizontal and another almost vertical. The vertical one corresponds to the slow solar wind $\left(V \approx 300-400 \mathrm{~km} \mathrm{~s}^{-1}\right)$, and the horizontal one to the fast solar wind ( $V>400 \mathrm{~km} \mathrm{~s}^{-1}$ and low values of $n$ and $B$ ). If fitted by the power-law, the relationships are given by $n=1.4 \times 10^{6} \times V^{-2.0 \pm 0.1}$, and $B=4710 \times V^{-1.1 \pm 0.1}$, with the correlation coefficients $R=0.69$ and $R=0.58$, respectively. Although the applied power-law fit is obviously more appropriate than the linear fit, it still shows a large deviation from the data in the slow-wind velocity range $\left(v<400 \mathrm{~km} \mathrm{~s}^{-1}\right)$. Thus, the obtained power-law relationships can be applied only to the fast solar wind.

We note that the first relationship, which can be expressed as $n V^{2}=$ const., resembles that of kinetic energy conservation. Similarly, the latter correlation (approximately $B V=$ const.) resembles to the magnetic flux conservation. However, we emphasize that these relationships cannot be interpreted in this way, since there is time lag between the adopted values of $V$ and $n$, as well as $V$ and $B$. These empirical forms reflect a complex/dynamical time-space relationship in the solar wind, that nevertheless produce a quite simple quantitative relationship. Although their meaning is not clear, they are certainly interesting, and deserve further analysis/interpretation from the theoretical point of view. 
G. Verbanac et al.: $\mathrm{CH}$, solar wind and their geoeffectiveness
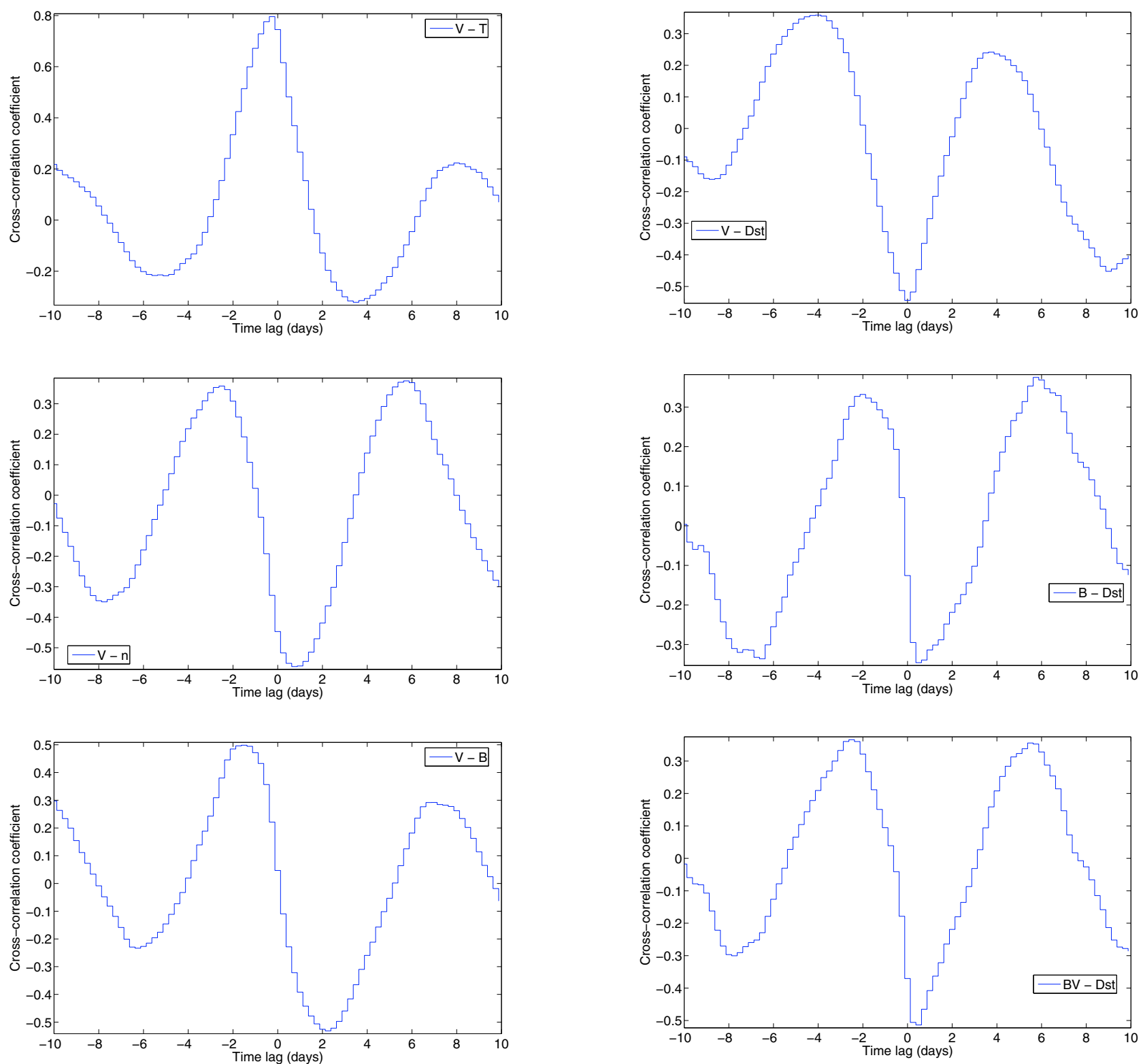

Fig. 9. Cross-correlation functions describing relationships between solar wind parameters $(V-T, V-n$, and $V-B)$.

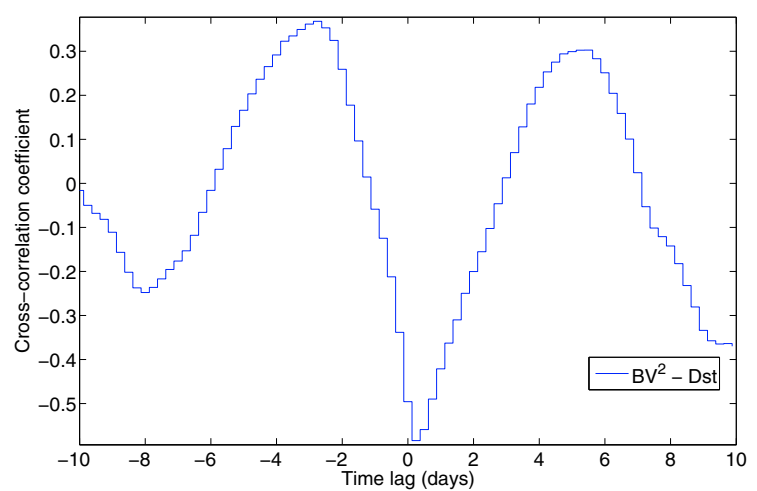

Fig. 10. Cross-correlation functions describing the relationship between the solar wind parameters and $D s t$ index $(V-D s t, B-D s t, B V-D s t$, and $B V^{2}-D s t$ ). 
A\&A 526, A20 (2011)
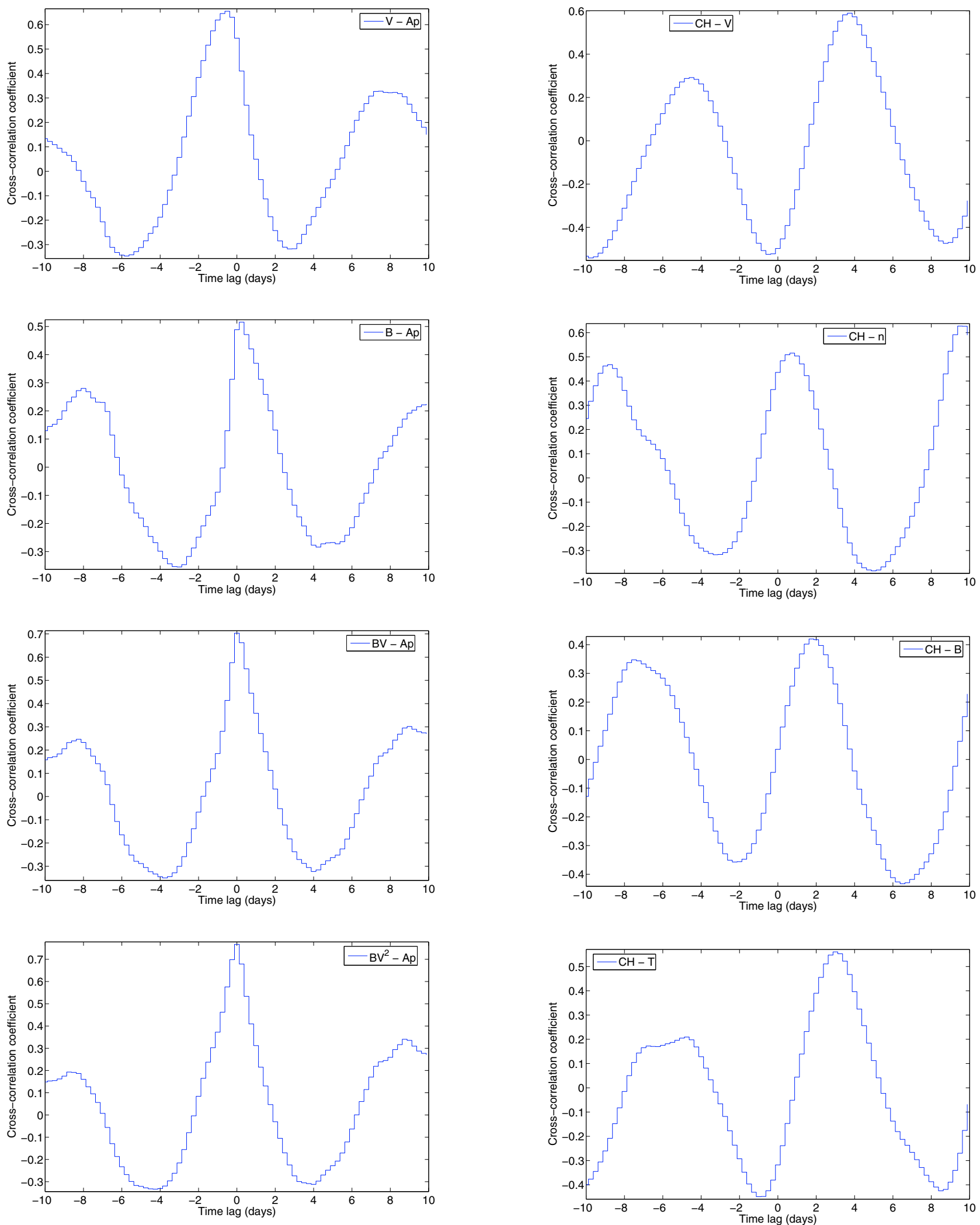

Fig. 11. Cross-correlation functions describing relationships between the solar wind parameters and $A p$ index $(V-A p, B-A p, B V-A p$, and $\left.B V^{2}-A p\right)$.

Fig. 12. Cross-correlation functions describing relationships between the coronal hole fractional areas $\mathrm{CH}$ and the solar wind parameters. 
G. Verbanac et al.: $\mathrm{CH}$, solar wind and their geoeffectiveness
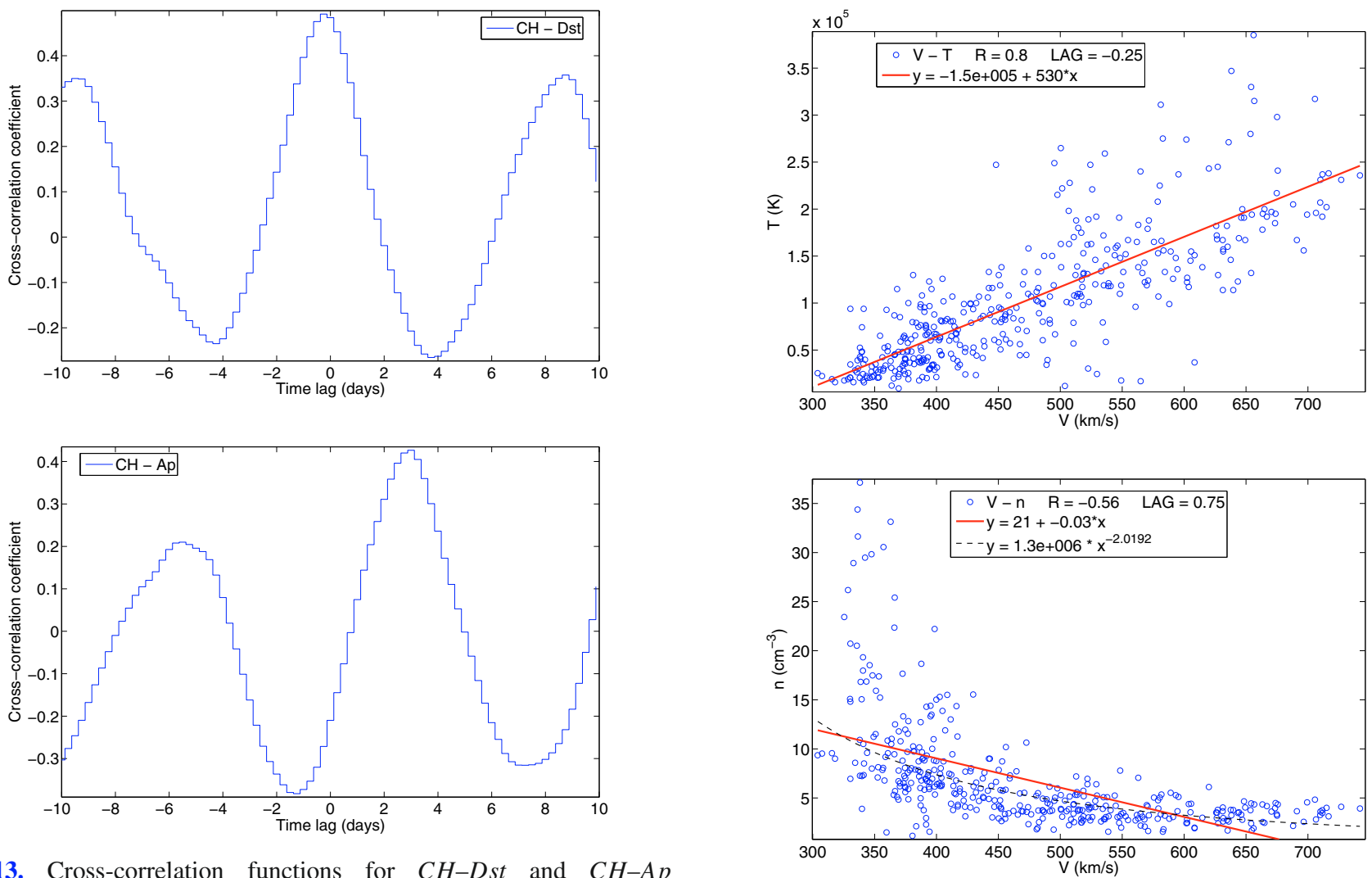

Fig. 13. Cross-correlation functions for $\mathrm{CH}-\mathrm{Dst}$ and $\mathrm{CH}-\mathrm{Ap}$ relationships.

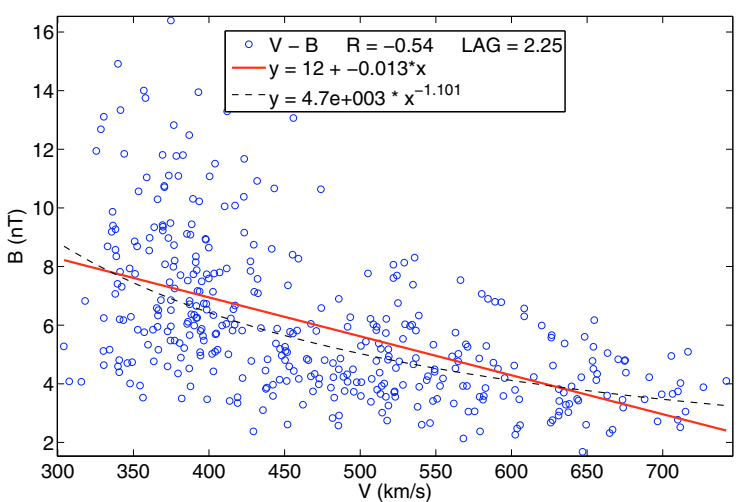

Fig. 14. Correlations $V-T, V-n$, and $V-B$. The linear least squares fits are shown by full lines and the power-law fits by dashed lines. The fit parameters, the correlation coefficient $R$, and the applied time lag $\Delta t$ are shown in the insets. 